\title{
Isotropically weighted intravoxel incoherent motion brain imaging
}

at $7 \mathrm{~T}$

\author{
Ivan I. Maximov ${ }^{\mathrm{a}, \mathrm{b}, *}$, Sebastian Vellmer ${ }^{\mathrm{c}, \mathrm{d}}$ \\ ${ }^{a}$ Department of Psychology, University of Oslo, Oslo, Norway \\ ${ }^{b}$ Norwegian Centre for Mental Disorders Research (NORMENT), KG Jebsen Centre for Psychosis \\ Research, Oslo University Hospital, Oslo, Norway and Institute of Clinical Medicine, University of Oslo, \\ Oslo, Norway \\ ${ }^{c}$ Bernstein Center for Computational Neuroscience, Berlin, Germany \\ ${ }^{d}$ Department of Physics, Humboldt University, Berlin, Germany
}

\begin{abstract}
Perfusion magnetic resonance imaging (MRI) is a promising non-invasive technique providing insights regarding the brain's microvascular architecture in vivo. The scalar perfusion metrics can be used for quantitative diagnostics of various brain abnormalities, in particular, in the stroke cases and tumours. However, conventional MRI-based perfusion approaches such as dynamic contrast-enhanced perfusion imaging or arterial spin labelling have a few weaknesses, for instance, contrast agent deposition, low signal-to-noise ratio, limited temporal and spatial resolution, and specific absorption rate constraints. As an alternative, the intravoxel incoherent motion (IVIM) approach exploits an extension of diffusion MRI in order to estimate perfusion parameters in the human brain. Application of IVIM imaging at ultra-high field MRI might employ the advantage of a higher signal-to-noise ratio, and thereby the use of higher spatial and temporal resolutions.

In the present work, we demonstrate an application of recently developed isotropic diffusion weighted sequences to the evaluation of IVIM parameters at an ultra-high 7T field. The used sequence exhibits high immunity to image degrading factors and allows one to acquire the data in a fast and efficient way. Utilising the bi-exponential fitting model of the signal attenuation, we performed an extensive analysis of the IVIM scalar metrics obtained by a isotropic diffusion weighted sequence in vivo and compared results with a conventional pulsed gradient sequence at $7 \mathrm{~T}$. In order to evaluate a possible metric bias originating from blood flows, we additionally used a truncated b-value protocol (b-values from 100 to $200 \mathrm{~s} / \mathrm{mm}^{2}$ with the step $20 \mathrm{~s} / \mathrm{mm}^{2}$ ) accompanied to the full range (b-values from 0 to 200 $\left.\mathrm{s} / \mathrm{mm}^{2}\right)$. The IVIM scalar metrics have been assessed and analysed together with a large and middle vessel density atlas of the human brain. We found that the diffusion coefficients and perfusion fractions of the voxels consisting of large and middle vessels have higher values in contrast to other tissues. Additionally, we did not find a strong dependence of the IVIM metrics on the density values of the vessel atlas. Perspectives and limitations of the developed isotropic diffusion weighted perfusion are presented and discussed.
\end{abstract}

Keywords: isotropically weighted diffusion, ultra-high field MRI, IVIM, perfusion 


\section{Introduction}

Perfusion magnetic resonance imaging (MRI) is an attractive clinical technique allowing one to perform a quantitative evaluation of many diseases in vivo: acute ischemic stroke [1], tumour diagnosis and prognosis [2, 3], diabetes and hyperintensity screening [4]. MRI provides an opportunity to determine perfusion metrics such as cerebral blood flow, cerebral blood volume, and mean transit time using several different approaches: an application of improved contrast mechanisms with external tracers (contrast agents); arterial spin labelling with arterial blood as endogenous tracer; and an adoption of diffusion weighted imaging considering the blood microcirculation as a pseudo-diffusion process in the randomly oriented microvessel network. Application of the first two methods has been shown to be clinically efficacious [5, 6]. However, improved contrast mechanisms and arterial spin labelling possess disadvantages in practice as well. Contrast agent boluses based on external contrast agents, for example, gadolinium, might lead to gadolinium deposition in the brain 7, 8. Furthermore, arterial spin labelling suffers from a low signal-to-noise ratio and a high specific absorption rate, in particular, at ultra-high fields [9, 10]. On the other hand, modification of the conventional diffusion MRI sequence assuming that the blood flow within microcapillary network can be represented as a complementary pseudo-diffusion process [11, 12], has found a wide range of applications for brain and body imaging [13, 14, 15, 16. This technique with low diffusion weightings is known as intravoxel incoherent motion (IVIM).

The diffusion phenomena in brain tissue is described by a broad range of the developed diffusion models [17, 18. In contrast, the IVIM theory and related mechanisms are still debated and demonstrate controversial results [19, 20, 21, 22]. Depending on the applied diffusion times, one can recall different mathematical models in fitting diffusion signal attenuation [20, 23. In the case of IVIM measurements, a bi-exponential fitting approach is a common step to separate diffusion fraction from the perfusion one along increasing diffusion weightings. However, it is well known that bi-exponential fitting is unstable [24, 25, 26]. Thus, IVIM demands an additional revision when modern achievements of MRI techniques are employed. The application of ultra-high field MRI can improve bi-exponential estimations due to the higher signal-to-noise ratio of the acquired data, in particular, in human brain imaging [27, 9]. In turn, the use of conventional diffusion weighting sequences might also be unoptimal, because it requires an application of at least three mutually orthogonal diffusion directions [27, and their sensitivity to susceptibility artefacts and subject motion [19, 28, 29]. In the case of many diffusion weights [30], the total measurement could be a time-consuming procedure. Besides, the necessity to consider the degrading imaging artefacts, such as susceptibility distortions, might play an important role in the case of microcapillary network anisotropy [29, 31], in particular, for high spatial resolution. Isotropic diffusion weighted sequences could avoid these obstacles and save expensive measurement time. Recently, isotropic weighting diffusion has obtained renewed interest due to its considerable practical impact [32], and the potential to adopt different diffusion models [32, 33]. A

\footnotetext{
${ }^{*}$ Corresponding author

Email address: ivan.maximov@psykologi.uio.no (Ivan I. Maximov)
} 
developed class of isotropic weighted diffusion sequences (FAst MEan Diffusion - FAMED) based on natural (co)sine modulations [34, 35] allows one to achieve several goals: a simple and straightforward implementation for human MRI scanners; a wide range of accessible diffusion weightings (so called $b$-values, including high $b$-values $>1500 \mathrm{~s} / \mathrm{mm}^{2}$ ); correct spatial isotropic averaging; accurate rotational invariants [34]; and robustness to the magnetic field imperfections, for example, susceptibility distortions [35].

In the present work, we would like to demonstrate advantages of the FAMEDcos sequence 34] for IVIM evaluation at ultra-high fields. We acquired three in vivo datasets using the developed sequence and estimated the correlations of the obtained perfusion metrics with a vessel density atlas [36] of large and middle sized vessels. To conclude, we discuss the strengths and limitations of the isotropic diffusion weighting sequences and the validity of the chosen IVIM bi-exponential fitting model for the human brain.

\section{Method and Materials}

\subsection{FAMEDcos sequences}

Within the range of clinically achievable diffusion times, the displacement of water molecules due to diffusion can be described by a Gaussian propagation function. A symmetric second-order diffusion tensor $\mathbf{D}$ allows one to represent diffusion signal attenuation $S$ in a form of mono-exponential decay:

$$
S=S_{0} \exp \left(-b \cdot \vec{n}^{T} \mathbf{D} \vec{n}\right),
$$

where $S_{0}$ is the signal without the diffusion weighting, $b$ is the applied diffusion weighting, and $\vec{n}$ is the unity vector, parallel to the applied diffusion gradient direction. In the case of pulsed field gradient (PFG) [37, diffusion encoding is performed by short gradient pulses $\vec{G}(t)$. Generally, when time-varying diffusion gradients $G(t)$ are applied, signal attenuation is formulated with help of the $\vec{q}(t)$-vector:

$$
S=S_{0} \exp \left(-\int_{0}^{t_{e}} \vec{q}^{T}(t) \mathbf{D}(t) \vec{q}(t) \mathrm{d} t\right),
$$

where $\vec{q}(t)=\gamma \int_{0}^{t} G(\tau) \mathrm{d} \tau$, and $\gamma$ is the gyromagnetic ratio and $t_{e}$ is the echo time (see pulse sequence scheme in Figure 11).

Selective time modulation of the diffusion encoding gradients allows one to suppress off-diagonal terms of the diffusion tensor $\mathbf{D}[34,35$, by satisfying the condition of mutual orthogonality:

$$
\int_{0}^{t_{e}} q_{i}(t) q_{j}(t) \mathrm{d} t=\frac{b}{3} \delta_{i j},
$$

where $\delta_{i i}=1$ is the Kronecker function. In turn, the signal attenuation of isotropically weighted diffusion is then:

$$
S=S_{0} \exp (-b \cdot \bar{D}),
$$

where $\bar{D}=\operatorname{Tr}(\mathbf{D}) / 3$ is the mean diffusivity. The simplest way to satisfy to Eq.(3) is to recall (co)sine modulations [34. 


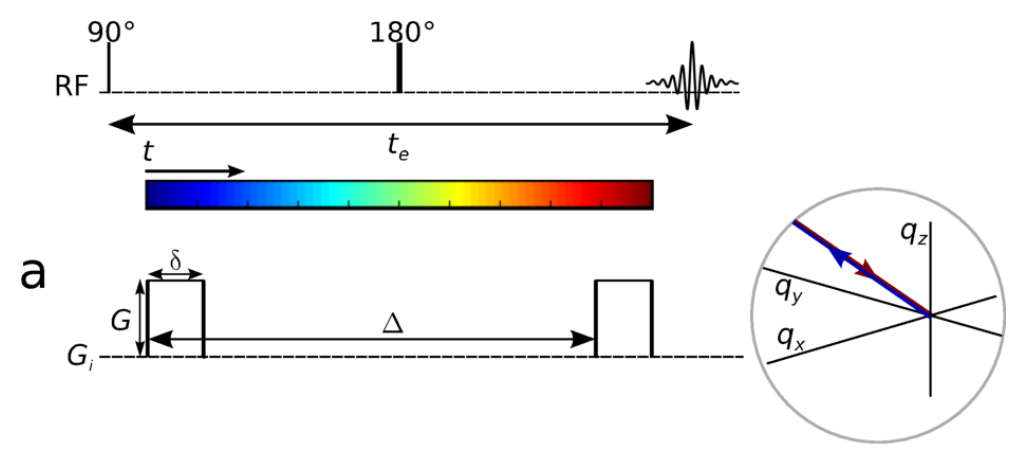

b

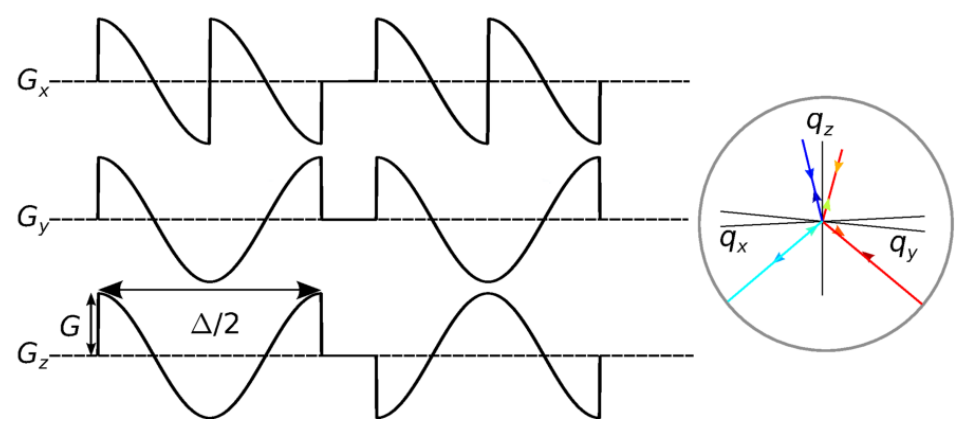

Figure 1: Schematic representation of diffusion weighted sequences. a) the conventional Stejskal-Tanner PFG pulse sequence with two short diffusion gradient $\delta$-pulses. Corresponding $q$-space trajectory is presented on the right side; $\mathbf{b}$ ) isotropical diffusion weighted FAMEDcos sequence with equal frequencies along each axes $x, y$, and $z$. The diffusion time in all sequences is $\Delta$.

The FAMEDcos sequence is presented by three orthogonal functions with the same frequency along the Cartesian axes:

$$
\vec{G}(t)=G \begin{cases}\left(\begin{array}{c}
\cos \left(\frac{4 \pi}{\Delta} t+\phi\right) \\
\cos \left(\frac{4 \pi}{\Delta} t\right) \\
\left.\cos \left(\frac{4 \pi}{\Delta} t+\theta\right)\right)
\end{array}\right), & \text { if } \quad 0<t \leq \frac{\Delta}{2}, \\
0, \quad \text { if } \quad \frac{\Delta}{2}<t \leq \frac{\Delta}{2}+t_{180}, \\
-\left(\begin{array}{c}
\cos \left(\frac{4 \pi}{\Delta}\left(t-t_{180}+\phi\right)\right) \\
\cos \left(\frac{4 \pi}{\Delta}\left(t-t_{180}\right)\right) \\
\left.\cos \left(\frac{4 \pi}{\Delta}\left(t-t_{180}+\theta\right)\right)\right)
\end{array}\right), & \text { if } \quad \frac{\Delta}{2}+t_{180}<t \leq \Delta+t_{180} .\end{cases}
$$

where phase shifts $\phi$ and $\theta$ are equal to

$$
\begin{aligned}
& \phi=\left\{\begin{array}{l}
\pi \text { if } \frac{\Delta}{4}<t<\frac{\Delta}{2} \\
\pi \text { if } \frac{3 \Delta}{4}+t_{180}<t<\Delta+t_{180} \\
0 \text { else }
\end{array}\right. \\
& \theta=\left\{\begin{array}{l}
\pi \text { if } \frac{\Delta}{2}+t_{180}<t<\Delta+t_{180} \\
0 \text { else }
\end{array}\right.
\end{aligned}
$$


Fig.1 b shows a schematic representation of FAMEDcos sequence used in the present work. The corresponding $b$-value is

$$
b_{\text {FAMEDcos }}=3 \int_{0}^{t_{e}} q_{i}(t) q_{i}(t) d t=G^{2} \gamma^{2} \frac{3 \Delta^{3}}{32 \pi^{2}} .
$$

\subsection{Imaging protocols}

All data were measured using a Siemens 7T MAGNETOM system (Siemens Medical Systems, Erlangen, Germany) equipped with a $70 \mathrm{mT} / \mathrm{m}$ gradient coil with maximal slew rate of $200 \mathrm{~T} / \mathrm{m} / \mathrm{s}$ and a 32 channel head coil. We measured three healthy male volunteers who gave written informed consent prior to participation. All volunteers had no self-reported history of any psychiatric or neurological diseases and did not suffer from any brain injuries. The study was approved by the local ethical committee.

Both diffusion weighted sequences PFG and FAMEDcos have been implemented into a custom spin-echo echo-planar imaging sequence. The imaging parameters were: field of view FoV $=216 \mathrm{~mm}$; spatial resolution $1.8 \times 1.8 \times 1.8 \mathrm{~mm}^{3}$; number of slices 40 ; repetition time $\mathrm{TR}=10 \mathrm{sec}$; echo time $\mathrm{TE}=110 \mathrm{~ms}$; number of measurements 4 (for FAMEDcos); partial Fourier factor 6/8; GRAPPA acceleration factor 2; readout bandwidth $\mathrm{BW}=2083 \mathrm{~Hz} / \mathrm{Px}$; diffusion time $\Delta=96 \mathrm{~ms}$; $b$-value range (FAMEDcos): 0, 20, 40, 60, 80, 100, 120, 140, 160, $180,200,500 \mathrm{~s} / \mathrm{mm}^{2}$; $b$-value range (PFG): 50, 100, 150, 200, $500 \mathrm{~s} / \mathrm{mm}^{2}$ and 6 non-coplanar diffusion encoding directions; acquisition time per protocol TA $=8: 20 / 5: 30 \mathrm{~min}$, correspondingly. Diffusion images with opposite phase encoding direction at $\mathrm{b}=0 \mathrm{~s} / \mathrm{mm}^{2}$ were acquired after FAMEDcos and PFG sequences. Additionally, $\mathrm{T}_{1}$-weighted MPRAGE images were acquired with the following parameters: $\mathrm{TE} / \mathrm{TR}=2.73 / 2500 \mathrm{~ms} ; \mathrm{FoV}=256 \mathrm{~mm}$; spatial resolution $1 \times 1 \times 1 \mathrm{~mm}^{3}$; GRAPPA acceleration factor 3 ; bandwidth $\mathrm{BW}=260 \mathrm{~Hz} / \mathrm{Px}$; and TA 2:59 min.

\subsection{Data post-processing and IVIM scalar metric estimation}

Before estimation of the IVIM scalar metrics, we performed typical post-processing steps for diffusion data preparation. In order to avoid noise-originated bias in the diffusion scalar metrics [38], we applied a principal component analysis-based approach for automatic removal of noise-specific components developed by Veraart et al. [39]. Next, we performed a Gibbs-ringing artefact correction using the approach developed by Kellner and colleagues [40]. Finally, we applied a correction of the artefacts originated from the head motion, eddycurrents and susceptibility induced distortions using the topup and eddy utilities from the FSL package [41]. In order to improve the estimation of scalar metrics, Gaussian smoothing with a kernel of $1 \times 1 \times 1 \mathrm{~mm}^{3}$ was applied. The brain mask used in the eddy artefact correction and metric estimations was extracted by the BET utility [42].

A main assumption proposed by Le Bihan and coworkers [11, 12] is that the blood flow within the microcapillary network captured by an imaging voxel can be represented by an analogue of the random walk process of the water molecules. A few variations of this model are presented in the literature [20, 27, 31], including use of high $b$-values [43] and three compartments model [44]. As bi-exponential fitting is frequently used in the 
modelling of different tissue types, we assume that a two-compartment model of IVIM is a good approximation:

$$
\frac{S}{S_{0}}=f \cdot \exp \left[-b \cdot\left(D^{*}+\bar{D}\right)\right]+(1-f) \cdot \exp [-b \cdot \bar{D}],
$$

where $f$ is the perfusion fraction, $\bar{D}$ is the conventional mean diffusion coefficient, and $D^{*}$ is the pseudo-diffusion coefficient representing the microcapillary random flows. It is well known that the bi-exponential fitting procedure is unstable [25, 45, 46]. To carefully extract the IVIM parameters from Eq. (9) we applied a three-step algorithm using an in-house Matlab script (The MathWork, Natick, USA):

- Initially, the estimation of the conventional diffusion coefficient $D$ was done by using a linearised Eq. (4) and $b$-values: $b=200$ and $500 \mathrm{~s} / \mathrm{mm}^{2}$;

- Next, for the fixed diffusion $D$ in Eq. (9), a constrained optimisation was used based on the interior-point algorithm [47]; the constraints are $D^{*} \subset[1 ; 50] \times 10^{-3} \mathrm{~mm}^{2} / \mathrm{s}$ and $f \subset\left[10^{-5} ; 0.3\right]$.

- If physically meaningful values could not be obtained from the previous step, we repeated the constrained optimisation algorithm based on the interior-point method including $S_{0}$ as an unknown variable.

The chosen approach of IVIM metric estimations has been shown to be efficient compared to other algorithms [48, 49]. Another estimation of IVIM metrics, in particular, in the case of low signal-to-noise ratio, can be implemented using Bayesian approaches [50, 51]. In our experimental setup the signal-to-noise ratio was high enough across all brain regions, i.e. it allows us to use a simple numerical implementation in contrast to widely popular Bayesian methods [27, 52]. To consider the influence of fast intra-voxel flows on the estimated IVIM metrics 23], we assumed that flow-based shifts in IVIM parameters are significant at very low $b$-values. If in the fitting procedure we reject the low $b$-values, namely below $100 \mathrm{~s} / \mathrm{mm}^{2}$, then we can decrease the influence of flow-based artefacts. Repeating the same analysis performed for the full $b$-value range we could estimate the influence of flow-based bias to the IVIM values. Below we used following notations: Protocol I is for the full range of $b$-values, and Protocol II is for the reduced $b$-value range that to demonstrate the analysis difference based on the flow compensation.

After the estimation of the IVIM metrics in diffusion space, we coregistered obtained scalar maps $\left(f-, D^{*}\right.$-, and $D$-images $)$ to the $T_{2}$-weighted Montreal Neurological Institute (MNI) template image using cubic spline interpolation. In order to find the most accurate matrix of transformation along the coregistration procedure, we used the $b=0 \mathrm{~s} / \mathrm{mm}^{2}$ image of the diffusion dataset and two-step coregistration. Firstly, the coregistration was performed using the FLIRT utility of the FSL package [53] with normalised mutual information as a criterion fit in the case of affine transformation. Then, we applied nonlinear transformation using the FNIRT utility of the FSL package [54]. For further segmentation, we used grey (GM) and white matter (WM) masks provided by the MNI atlas. Additionally, 

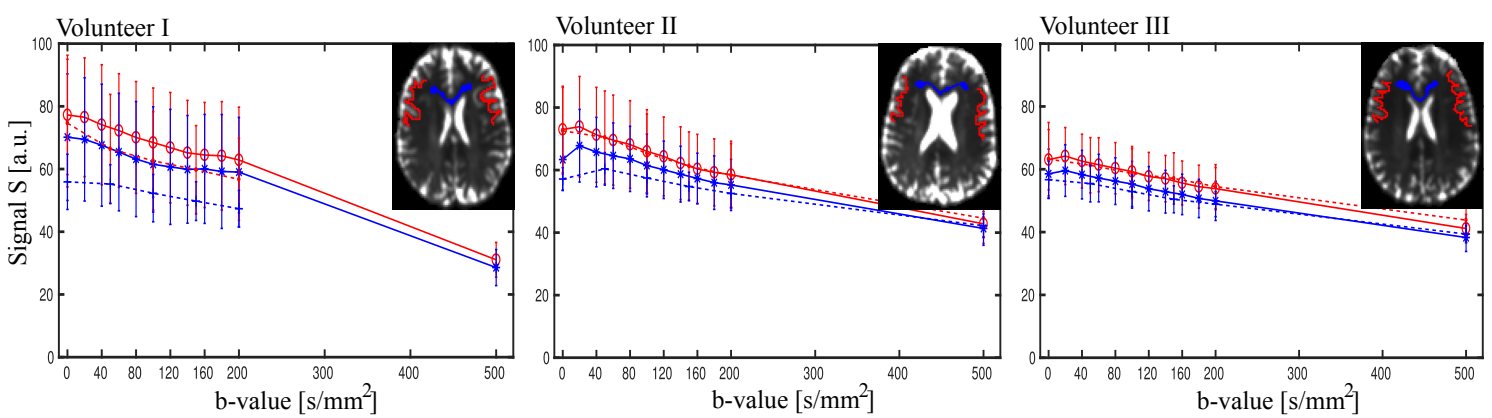

Figure 2: Averaged signal decays after performed post-processing corrections of the data for three volunteers. Two different regions are manually chosen in grey and white (red/blue, respectively) matter for each volunteer. The results are presented for both pulse sequences FAMEDcos (solid line) and PFG (dashed line).

we used the freely accessible brain vessel atlas [36 aligned in the MNI space. Afterwards, all coregistration steps were visually inspected in order to detect any misalignment.

\section{Results}

The signal decays averaged over different regions of interest are presented in Figure2, The GM and WM regions with signal decays were manually chosen for three volunteers. To provide comparable results among the subjects we chose for WM a corpus callosum region and for GM a region of cerebral cortex located over left and right Broca's areas.

Figure 3 shows example slices of in vivo IVIM metrics obtained by the bi-exponential fitting in Eq. (9): diffusion coefficient $(D)$, perfusion fraction $(f)$, and pseudo-diffusion coefficient $\left(D^{*}\right)$ metrics. The pseudo-diffusion coefficients and perfusion fractions are presented for Protocols I and II, in order to demonstrate the quantitative difference between estimated IVIM metrics produced by full and flow compensated $b$-value ranges. A quantitative parametrisation of the metric distribution is accompanied by the corresponding histograms. Data for other volunteers are presented in the Supplementary Materials (Figure S1).

In Figure 4, we presented correlations between diffusion coefficients obtained from FAMEDcos and PFG sequences (Figs 4 a) and IVIM metrics from Protocol I and II (Fig.4 c) for one volunteer. For each scatter plot, we computed the Pearson correlation coefficient between metrics derived from FAMEDcos and PFG data and between Protocols I and II, respectively. In order to eliminate the influence of coil sensitivity on the estimated metric, we chose 60 axial slices of the brain. Further statistical analysis was based on the IVIM metrics averaged over each axial slice in accordance with chosen masks such as WM or GM MNI masks. The slice position is encoded by the colour map (see Fig. $4 \mathrm{~b}$ ). This type of slice metric averaging was used in the next section of results as well.

Figure5 presents correlations between IVIM scalar metrics derived from FAMEDcos data and vessel density atlas for voxels from GM and WM. Similar to Fig. 4, we performed slice averaging metrics for scalar metrics and vessel density map. In order to decrease the influence of the slice metric variability, we applied a robust regression algorithm using the 

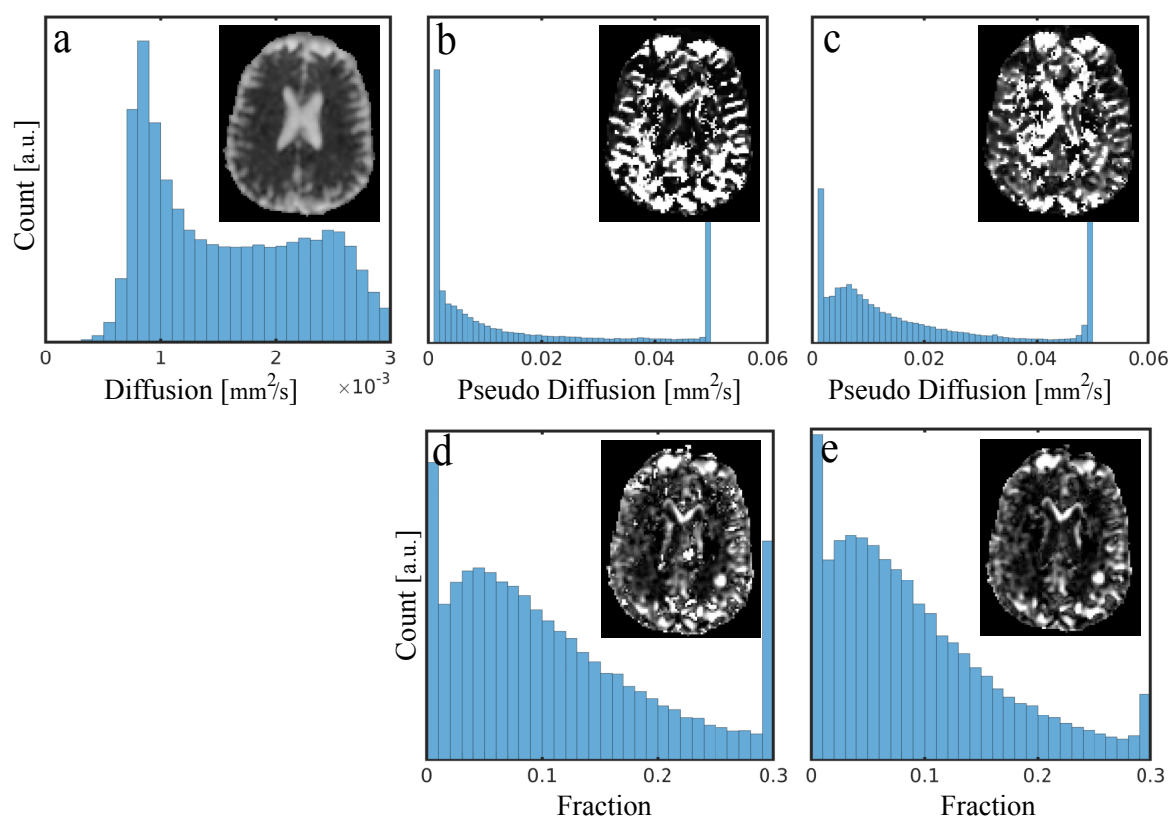

Figure 3: An example of estimated IVIM scalar metrics using FAMEDcos sequence and two Protocols I $(\mathbf{b}, \mathbf{d})$ and II $(\mathbf{c}, \mathbf{e})$. We manually chose one slice and plotted its metric histograms: a) ordinary diffusion coefficients; b,c) pseudo-diffusion coefficients; $\mathbf{d}, \mathbf{e})$ perfusion fractions.
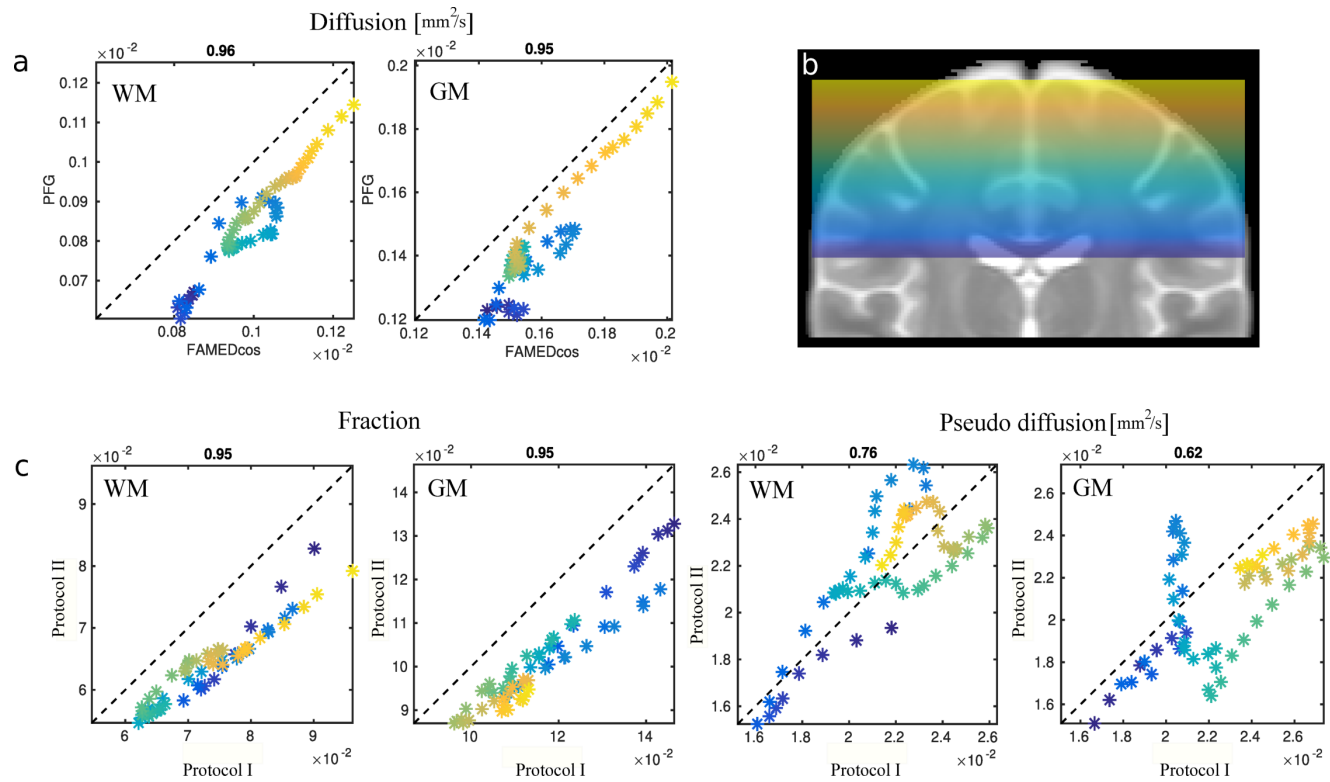

Figure 4: Scatter plots of the diffusion coefficients between FAMEDcos and PFG sequences (a) and IVIM metrics obtained by two Protocols I and II (c). The colours encode axial slice positions for slice-averaged values (b). The dashed black line corresponds to the identity line. The slice averaging is performed using GM and WM masks. The Pearson correlation coefficients are presented on top of each scatter plot frame. 

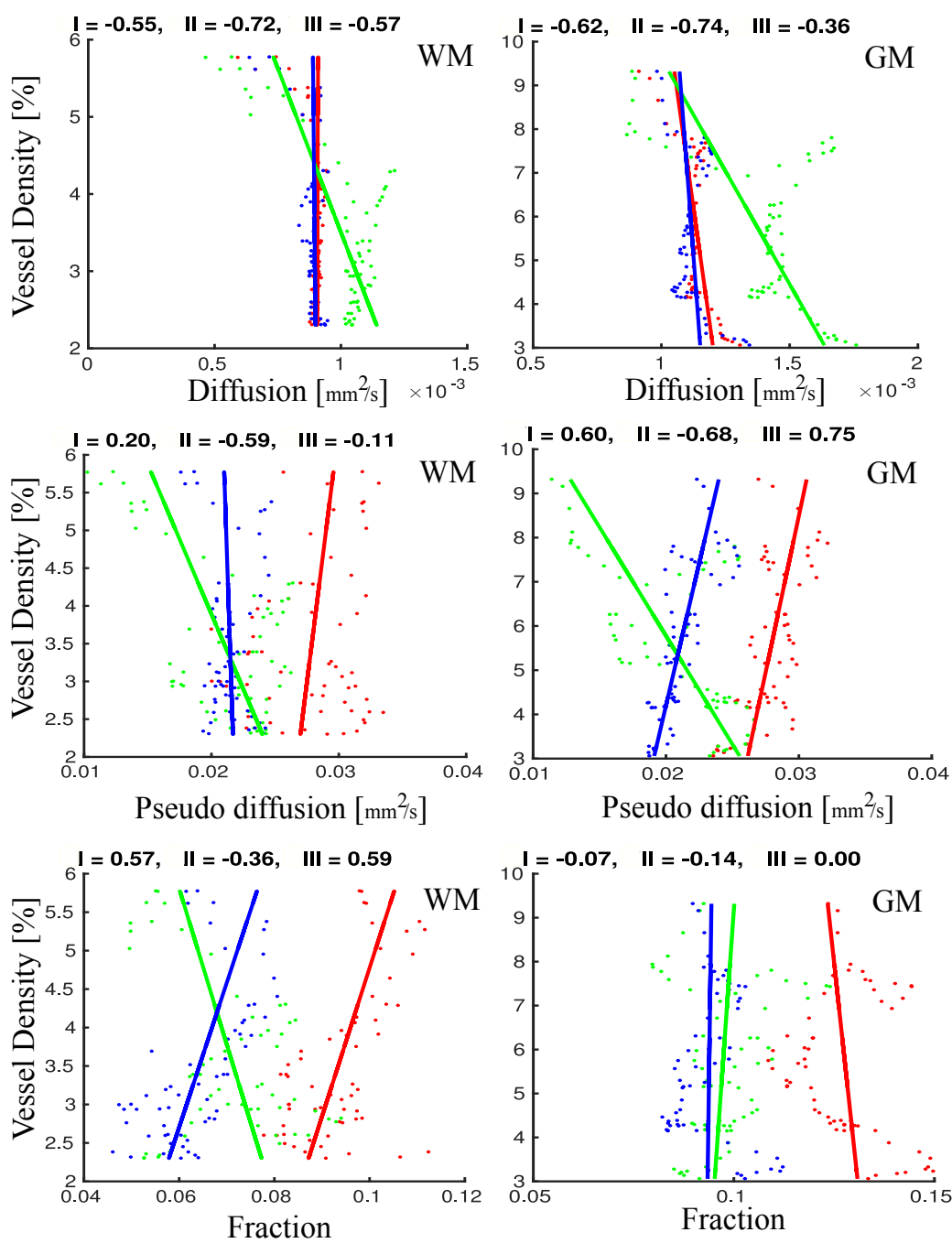

\section{Volunteer I \\ Volunteer II \\ Volunteer III}

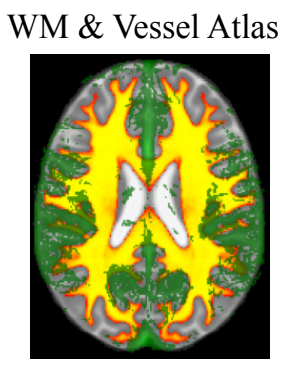

\section{GM \& Vessel Atlas}

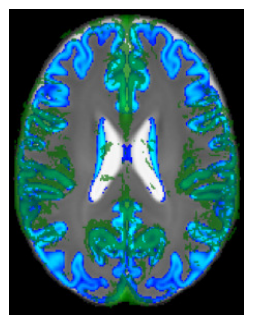

Figure 5: Correlation of IVIM scalar metrics and vessel density atlas for WM (left) and GM (right). For each volunteer (I, II, and III, respectively) we evaluated the Pearson correlation coefficients and fitted the data dependence using a robust regression algorithm.

robustfit-function of Matlab. The robust fitting is plotted as lines for each metric taking into account Protocol II only. Similar results for Protocol I are presented in the Supplementary Materials (Fig. S2).

To assess a possible influence of large and middle vessels on the IVIM scalar metrics, namely, the perfusion fraction, pseudo-diffusion coefficient and conventional diffusion coefficient, we built two characteristic datasets for GM and WM regions: we superposed the GM or MW masks with the vessel atlas [36] and marked coincided metrics (so-called in-mask) and metrics located in GM or WM, but not in the atlas (so-called out-mask). The examples of in-mask and out-mask values are presented in Figure6. The resulting scatter plots for Protocol II and slice averaged metrics are presented in Fig.6 for three volunteers. Similar results for Protocol I are presented in the Supplementary Materials (see Fig. S3). 

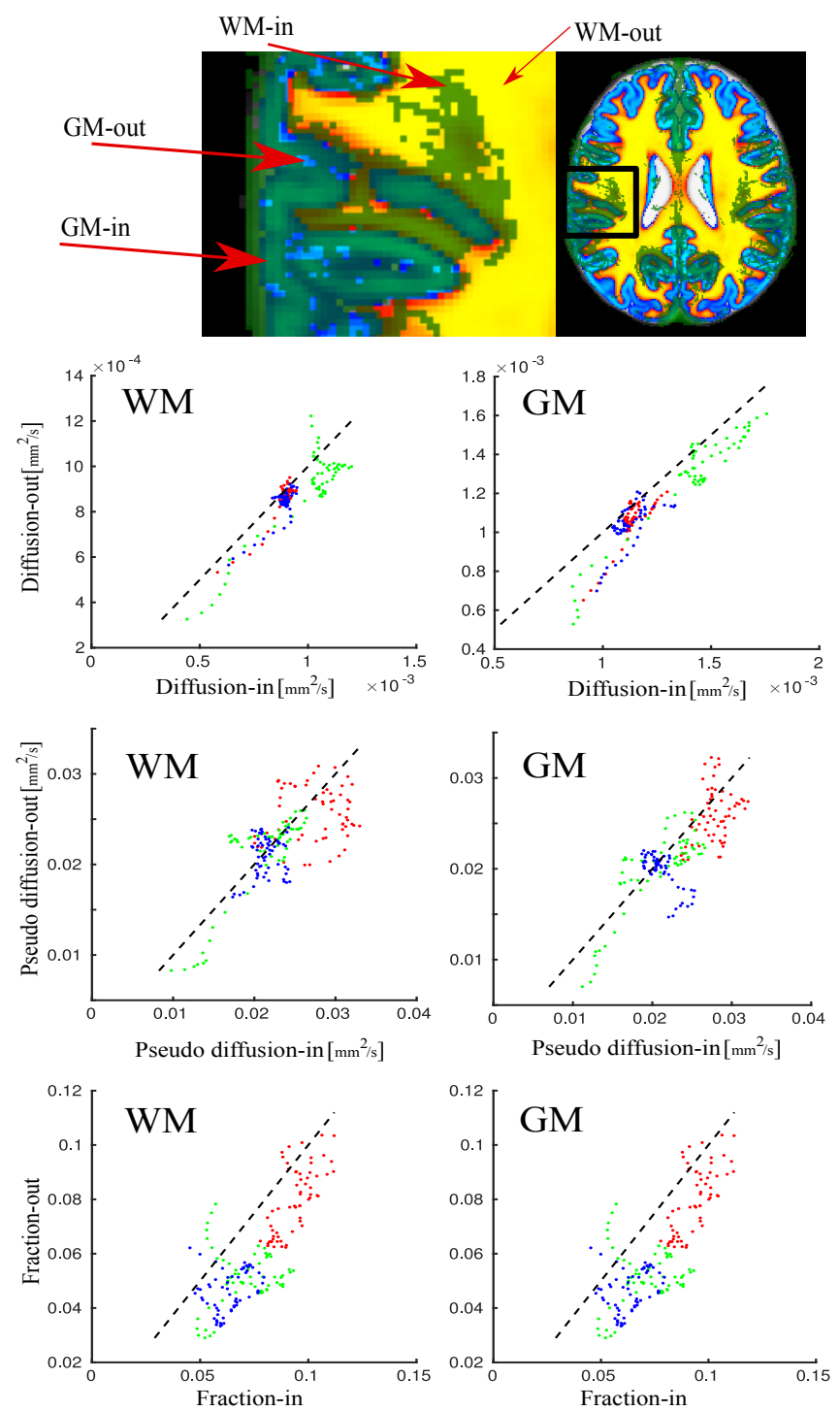

Figure 6: Scatter plots between the IVIM scalar metrics covaried with large and middle vessel locations in the brain. The vessel atlas is produced by Viviani [36. Two data groups were used by a conjunction of GM and WM masks with the vessel atlas: in-mask values belong to both spaces: GM (blue-light blue) or WM (yellow-red) and the vessel atlas (green); out-mask values belong only to GM or WM regions, but not to the vessel atlas. Data are presented for Protocol II. 
In order to provide a statistical comparison between in- and out-mask metrics in Fig.6 we applied Mann-Whitney U-tests for all subjects. The Mann-Whitney U-test allows one to compare mean values from two datasets without an assumption that the data are normally distributed. The statistical comparisons were performed using Matlab function ranksum. For diffusion coefficients in WM, the in-mask values are higher with $\mathrm{p}<\left(6 \cdot 10^{-7} ; 2 \cdot 10^{-7} ; 3 \cdot 10^{-9}\right)$ for I, II and III volunteers, respectively. In the case of GM, the diffusion coefficients are higher in in-mask area with $\mathrm{p}<\left(3 \cdot 10^{-6} ; 4 \cdot 10^{-4} ; 5 \cdot 10^{-6}\right)$. For pseudo-diffusion coefficients in WM, the in-mask values are significantly higher for one volunteer only; for GM case, two volunteers exhibited the higher pseudo-diffusion values in the in-mask area (see Fig. 66. However, the perfusion fractions for WM and GM in-mask values are significantly higher for all participants with $\mathrm{p}<\left(2 \cdot 10^{-9} ; 3 \cdot 10^{-13} ; 1 \cdot 10^{-16}\right)$ and $\mathrm{p}<\left(6 \cdot 10^{-6} ; 5 \cdot 10^{-3} ; 2 \cdot 10^{-4}\right)$, respectively.

\section{Discussion}

The scalar perfusion metrics based on IVIM approach still attract researchers' interest. One reason for this is the absence of a commonly accepted IVIM perfusion model in the human brain explaining and predicting the microcapillary architecture from measured signal attenuation. Therefore, an additional theoretical description of the perfusion processes for IVIM and novel experimental facts are needed and might shed light on the problem. Research and clinical IVIM protocols frequently require the acquisition of at least three orthogonal diffusion directions with multiple $b$-values in order to accurately evaluate the bi-exponential signal decomposition, taking into account repeated measurements that to increase the signal-to-noise ratio and avoid an influence of possible imaging artefacts. Such an approach has clear limitation in time, in particular, at ultra-high field MRI where the magnetic field exposure and specific absorption rate constraint [55] can be a problem. An increasing spatial resolution of conventional diffusion MRI brings additional confounds by the increased influence of susceptibility effects to the correct choice of the diffusion gradient directions. Thus, single-shot imaging techniques such as isotropic diffusion weighted sequences [34, 35] might offer a solution. In turn, isotropic diffusion weighted sequences should possess a few important features: simple scanner-based implementation, robustness to eddy-currents and susceptibility-based distortions, a wide spectrum of the accessible $b$ values and diffusion times, and to demonstrate reliable rotational invariant metrics [56]. Recently proposed pulse sequences based on original (co)sine modulations allowed us to achieve the above mentioned features [34, 35]. As a result, we demonstrated a proficiency of the application of isotropic diffusion weighted sequences for in vivo perfusion measurements in the human brain at ultra-high field MRI.

A quantitative comparison of the results between developed sequence FAMEDcos and conventional PFG demonstrated good mutual agreement. We found a high correlation between mean diffusivities obtained by both sequences (see Fig.4 4). Interestingly, the linear correlations are very good for both GM and WM areas. The averaged diffusion coefficients are increasing along the axial slice shift from the corpus callosum region up to the top axial cortical slices. However, centres of mass of data distributions for WM and GM are localised 
at the well-known mean values: $0.9 \times 10^{3} \mathrm{~mm}^{2} / \mathrm{ms}$ and $1.5 \times 10^{3} \mathrm{~mm}^{2} / \mathrm{ms}$, respectively (see also Fig. 3). Though, the FAMEDcos diffusion coefficients are in feasible range, the correlations gave higher FAMEDcos values in comparison to the PFG metrics. This discrepancy may have originated from unoptimal selection of PFG diffusion directions and requires additional tests with a more accurately established PFG/FAMEDcos protocol at 7T. At the same time, conventional diffusion coefficients were estimated from quite small $b$-values (200 and $500 \mathrm{~s} / \mathrm{mm}^{2}$ ), which might have led to the biased estimations, i.e. use of higher $b$-values could improve the assessment.

Analysis based on the flow-compensated Protocol II demonstrated the same behaviour of IVIM metrics as in the case of Protocol I (see Fig. 3). We applied the FAMEDcos sequence in the so-called diffusion limit [27], when the intricate effect of the perfusion processes should be negligible [20, 31]. It is worth mentioning, that the isotropic character of diffusion weightings in the applied sequences allowed us to avoid a possible effect of perfusion anisotropy [31, 29] at the chosen spatial resolution. Consequently, it allowed us to work with correctly isotropic averaged metrics of microcapillary networks. At the same time, our hypothesis about the flow compensation by shortened $b$-value range found a reasonable confirmation (see Figs. 3 and 4c). The perfusion fraction in both cases for Protocol I and II possessed very high correlations, but in the case of Protocol II the fraction metrics are decreased for WM and GM. On the contrary, the pseudo-diffusion values are almost linearly correlated for WM, and close to such dependence in the case of GM. Thus, the shortened $b$-value range could be a good approximation of flow compensated sequences.

From brain anatomy it is known that the cortical GM regions are a highly vascularisated medium [57]. The fraction of both large and middle veins and arteries is reducing along to the deeper brain structures [36, 58]. Besides that, an effect of the reducing microcapillary network and its density along the main vessels for WM/GM should be detectable by the IVIM approach as well. In order to test our hypothesis that IVIM scalar metrics might correlate with the large and middle vessel density and location, we separated the scalar metrics into two groups in GM and WM regions: crossed with anatomical masks (segmented GM or WM) and vessel atlas and inverted regions, i.e. in-mask and out-mask metrics, respectively (see Fig.66). We assumed that regions with a high vessel density should possess dense microcapillary networks as well. As a consequence, higher perfusion fractions can be detected and localised. A reasonable verification of this hypothesis can be the same statistical behaviour of the flow-compensated Protocol II as well. This statistical comparison is presented in Fig.6. We can claim that GM regions fully support our idea and perfusion fractions (proportional to the microcapillary density) are higher for in-mask areas, in particular, for Protocol II case. A similar effect is detectable for the WM regions. Surprisingly, the pseudo-diffusion coefficients show an alternating behaviour depending on the volunteer. However, this effect can be explained by lower reliability of the pseudo-diffusion coefficient estimations due to bi-exponential fitting [19]. Interestingly, the presence of large and middle vessels influenced the conventional diffusion coefficient as well (see Fig.6), in particular, the regions with grey matter. The significant difference of diffusion coefficients between in- and out-masks might be a sign for additional effect influencing on the DTI metrics, such as perivascular space with corresponding convection processes [59]. 
In order to validate our hypothesis that the presence of vessels biases scalar metrics, we evaluated the direct correlation between scalar metrics and a vessel density atlas (see Fig. 5). It is clear that conventional diffusion metrics are independent of the vessel density. In turn, the same results are obtained for the perfusion fraction in GM, in particular, using Protocol II and pseudo-diffusion coefficient in WM. Non-significant variations of IVIM metrics can be observed for other cases. Thus, we can conclude that the vessel density itself does not correlate with IVIM metrics.

In our opinion, the strong correlations between the vessel density atlas and the perfusion fraction allows one to perform an indirect estimation of the microcapillary architecture, i.e. microcapillaries are concentrated around middle and large vessels. Besides this, we can assert that the density vessel atlas might be a helpful additional variable in perfusion studies, in particular, for a neuronal activation problem and functional MRI due to the relation between the cerebral blood flow and neuronal density [60]. In fact, we assume that IVIM demonstrates only a qualitative perfusion effect but the estimated IVIM scalar metrics have the considerable variations depending on the subject or applied evaluation algorithm [61]. For example, an attempt to estimate the cerebral blood flow [12, 62, 13] led to the overestimated values in the studies comparing to other methods. Another possible reason for IVIM metric variability is the recently introduced paravascular space [63] where the convection lymphatic processes are slower compared to the vessel blood flows and might influence on IVIM scalar metrics. Thus, IVIM might be sensitive to additional extra-cellular space covarying with large and middle vessels. As a consequence, a more accurate mathematical model of signal attenuation in the case of IVIM is needed.

\subsection{Limitations}

Possible limitations of the present results are the low number of the subjects and coarse spatial resolution. Increasing the number of subjects should allow us to perform an accurate statistical analysis based on specific anatomical regions, for example, to analyse the difference between deep and cortical brain structures in order to support a hypothesis of importance of large and middle vessels as a possible IVIM covariance. At the same time, increasing spatial resolution should introduce an effect of microcapillary anisotropy and its influence to the IVIM signal, for instance, applying reduced field of view methods using optimal control algorithms [64, 65]. It is, in particular, important due to a presence of partial volume effect with cerebrospinal fluids. The voxels with significant percentage of CSF might introduce a significant bias in IVIM metric estimation due to problematic bi-exponential fitting [44. Another confounding effect could be relaxation time, namely, $\mathrm{T}_{2}$ which shortens along the increasing magnetic field. Thus, the pulse sequence with reduced echo time might improve the situation. Last but not least, we need to perform an accurate and exact validation of flowbased compensations using a reduced $b$-value range with correctly implemented gradientbased approaches [23].

\section{Conclusion}

This study demonstrates an application of isotropic diffusion weighted IVIM scalar metrics in brain perfusion imaging at ultra-high magnetic fields. Advantages of the obtained 
IVIM scalar metrics include higher signal-to-noise ratio, a very fast measurement procedure, and suppression of possible anisotropic contributions of microcapillary network. As a result, we found a high correlation of large and middle vessel density with IVIM metrics and conventional diffusion coefficients in the human brain. We anticipate that our isotropic diffusion weighted approach can find applications in other imaging areas such as breast and abdomenal MRI, or in other cases where a rapid acquisition is a decisive point, for instance, fMRI or hyperpolasrisation measurements.

\section{Acknowledgement}

Authors are very grateful to Dr. Rüdiger Stirnberg, Dr. Eberhardt D. Pracht, and Prof. Tony Stöcker for the access to $7 \mathrm{~T}$ facility at the German Center of Neurodegenerative Diseases (DZNE), Bonn, Germany, and assistance during the measurements. IIM gratefully thanks Dr. Daniel S. Quintana for his proofreading of the manuscript and UiO:Life Science fund for support of the work.

\section{References}

[1] J. M. Olivot, Imaging of brain ischemia, Revue Neurologique 167 (2011) 873-880.

[2] M. Law, S. Yang, H. Wang, J. S. Babb, G. Johnson, C. Chad, E. A. Knopp, D. Zagzag, Glioma grading: Sensitivity, specificity, and predictive values of perfusion MR imaging and proton MR spectroscopic imaging compared with conventional MR imaging, American Journal of Neuroradiology 24 (2003) 1989-1998.

[3] M. Law, R. J. Young, J. S. Babb, N. Peccerelli, S. Chheang, M. L. Gruber, D. C. Miller, J. G. Golfinos, D. Zagzag, G. Johnson, Gliomas: Predicting time to progression or survival with cerebral blood volume measurements at dynamic susceptibility-weighted contrast-enhanced perfusion MR imaging, Radiology 247 (2008) 490-498.

[4] S. Bal, M. Goyal, E. Smith, A. M. Demchuk, Central nervous system imaging in diabetic cerebrovascular diseases and white matter hyperintensities, Handbook of Clinical Neurology 126 (2014) 291-315.

[5] C. C. Quarles, L. C. Bell, A. M. Stokes, Imaging vascular and hemodynamic features of the brain using dynamic susceptibility contrast and dynamic contrast enhanced MRI, NeuroImage.

[6] X. Golay, M. Guenther, Arterial spin labelling: final steps to make it a clinical reality, Magnetic Resonance Materials in Physics, Biology and Medicine 25 (2012) 79-82.

[7] N. Murata, K. Murata, L. F. Gonzalez-Cuyar, K. R. Maravilla, Gadolinium tissue deposition in brain and bone, Magnetic Resonance Imaging 34 (2016) 1359-1365.

[8] A. Radbruch, Are some agents less likely to deposit gadolinium in the brain?, Magnetic Resonance Imaging 34 (2016) 1351-1354.

[9] Y. Wang, S. Moeller, X. Li, A. T. Vu, K. Krasileva, K. Ugurbil, E. Yacoub, D. J. Wang, Simultaneous multi-slice Turbo-FLASH imaging with CAIPIRINHA for whole brain distortion-free pseudo-continuous arterial spin labeling at 3 and 7T, NeuroImage 113 (2015) 279-288.

[10] E. Boudes, G. Gilbert, I. R. Leppert, X. Tan, G. B. Pike, C. Saint-Martin, P. Wintermark, Measurement of brain perfusion in newborns: pulsed arterial spin labeling (PASL) versus pseudo-continuous arterial spin labeling (pCASL), NeuroImage Clinical 6 (2014) 126-133.

[11] D. Le Bihan, E. Breton, D. Lallemand, L. M. Aubin, J. Vagnaud, M. Laval-Jeantet, Separation of diffusion and perfusion in intravoxel incoherent motion MR imaging, Radiology 168 (1988) 497-505.

[12] D. Le Bihan, R. Turner, The capillary network: A link between IVIM and classical perfusion, Magnetic Resonance in Medicine 27 (1992) 171-178. 
[13] C. Federau, K. OBrien, R. Meuli, P. Hagmann, P. Maeder, Measuring brain perfusion with intravoxel incoherent motion (IVIM): Initial clinical experience, Journal of Magnetic Resonance Imaging 39 (2014) 624-632.

[14] S. Suo, M. Cao, W. Zhu, L. Li, J. Li, F. Shen, J. Zu, Z. Zhou, Z. Zhuang, J. Qu, Z. Chen, J. Xu, Stroke assessment with intravoxel incoherent motion diffusion-weighted MRI, NMR in Biomedicine 29 (2016) $320-328$.

[15] P. X. Lu, H. Huang, J. Yuan, F. Zhao, Z. Y. Chen, Q. Zhang, A. T. Ahuja, B. P. Zhou, Y. X. J. Wang, Decreases in molecular diffusion, perfusion fraction and perfusion-related diffusion in fibrotic livers: A prospective clinical intravoxel incoherent motion MR imaging study, PLOS One 9 (2014) e113846.

[16] Y. Pang, B. Turkbey, M. Bernardo, J. Kruecker, S. Kadoury, M. J. Merino, B. J. Wood, P. A. Pinto, P. L. Choyke, Intravoxel incoherent motion (IVIM) MR imaging for prostate cancer: An evaluation of perfusion fraction and diffusion coefficient derived from different b-value combinations, Magnetic Resonance in Medicine 69 (2013) 553-562.

[17] V. G. Kiselev, Fundamentals of diffusion MRI physics, NMR in Biomedicine 30 (2017) e3602.

[18] D. S. Novikov, S. N. Jespersen, V. G. Kiselev, E. Fieremans, Quantifying brain microstructure with diffusion MRI: Theory and parameter estimation, arXive.org physics.bio-ph (2016) 1612.02059v1.

[19] C. Federau, Intravoxel incoherent motion MRI as a means to measure in vivo perfusion: A review of the evidence, NMR in Biomedicine 30 (2017) e3780.

[20] G. Fournet, J. R. Li, A. M. Cerjanic, B. P. Sutton, L. Ciobanu, D. Le Bihan, A two-pool model to describe the IVIM cerebral perfusion, Journal of Cerebral Blood Flow and Metabolism 37 (2017) $2987-3000$.

[21] J. J. Neil, J. J. H. Ackerman, Detection of pseudodiffusion in rat brain following blood substitution with perfluorocarbon, Journal of Magnetic Resonance 97 (1992) 194-201.

[22] R. M. Henkelman, J. J. Neil, Q. S. Xiang, A quantitative interpretation of ivim measurements of vascular perfusion in the rat brain, Magnetic Resonance in Medicine 32 (1994) 464-469.

[23] A. Ahlgren, L. Knutsson, R. Wirestam, M. Nilsson, F. Ståhlberg, D. Topgaard, S. Lasic, Quantification of microcirculatory parameters by joint analysis of flow-compensated and non-flow-compensated intravoxel incoherent motion (IVIM) data, NMR in Biomedicine 29 (2016) 640-649.

[24] J. Pekar, C. T. W. Moonen, P. C. M. van Zijl, On the precision of diffusion/perfusion imaging by gradient sensitization, Magnetic Resonance in Medicine 23 (1992) 122-129.

[25] A. A. Istratov, O. F. Vyvenko, Exponential analysis in physical phenomena, Review of scientific instruments 70 (1999) 1233-1257.

[26] F. Grinberg, I. I. Maximov, E. Farrher, N. J. Shah, Microstructure-informed slow diffusion tractography in humans enhances visualisation of fibre pathways, Magnetic Resonance Imaging 45 (2018) 7-17.

[27] A. S. Rydhög, M. J. van Osch, E. Lindgren, M. Nilsson, J. Lätt, F. Ståhlberg, R. Wirestam, L. Knutsson, Intravoxel incoherent motion (IVIM) imaging at different magnetic field strengths: What is feasible?, Magnetic Resonance Imaging 32 (2014) 1247-1258.

[28] V. G. Kiselev, Effect of magnetic field gradients induced by microvasculature on NMR measurements of molecular self-diffusion in biological tissues, Journal of Magnetic Resonance 170 (2004) 228-235.

[29] P. Kennel, C. Fonta, R. Guibert, F. Plourabou, Analysis of vascular homogeneity and anisotropy on high-resolution primate brain imaging, Human Brain Mapping 152 (2017) 340-347.

[30] A. Lemke, B. Stieltjes, L. R. Schad, F. B. Laun, Toward an optimal distribution of b values for intravoxel incoherent motion imaging, Magnetic Resonance Imaging 29 (2011) 766-776.

[31] T. Finkenstaedt, M. Klarhoefer, C. Eberhardt, A. S. Becker, G. Andreisek, A. Boss, C. Rossi, The IVIM signal in the healthy cerebral gray matter: a play of spherical and non-spherical components, NeuroImage 152 (2017) 340-347.

[32] C. F. Westin, H. Knutsson, O. Pasternak, F. Szczepankiewicz, E. Özarslan, D. van Westen, C. Mattisson, M. Bogren, L. J. O’Donnell, M. Kubicki, D. Topgaard, M. Nilsson, Q-space trajectory imaging for multidimensional diffusion MRI of the human brain, NeuroImage 135 (2016) 345-362.

[33] S. Lasic, F. Szczepankiewicz, S. Eriksson, M. Nilsson, D. Topgaard, Microanisotropy imaging quantification of microscopic diffusion anisotropy and orientational order parameter by diffusion MRI with 
magic-angle spinning of the q-vector, Frontiers in Physics 2 (2014) 11.

[34] S. Vellmer, R. Stirnberg, D. Edelhoff, D. Suter, T. Stöcker, I. I. Maximov, Comparative analysis of isotropic diffusion weighted imaging sequences, Journal of Magnetic Resonance 275 (2017) 137-147.

[35] S. Vellmer, D. Edelhoff, D. Suter, I. I. Maximov, Anisotropic diffusion phantoms based on microcapillaries, Journal of Magnetic Resonance 279 (2017) 1-10.

[36] R. Viviani, A digital atlas of middle to large brain vessels and their relation to cortical and subcortical structures, Frontiers in Neuroanatomy 10 (2016) 12.

[37] E. O. Stejskal, J. E. Tanner, Spin diffusion measurements: spin echoes in the presence of a timedependent field gradient, Journal of Chemical Physics 42 (1965) 288-292.

[38] E. D. Andre, F. Grinberg, E. Farrher, I. I. Maximov, N. J. Shah, C. Meyer, M. Jaspar, V. Muto, C. Phillips, E. Balteau, Influence of noise correction on intra- and inter-subject variability of quantitative metrics in diffusion kurtosis imaging, PLoS One 9 (2014) e94531.

[39] J. Veraart, E. Fieremans, D. S. Novikov, Diffusion MRI noise mapping using random matrix theory, Magnetic Resonance in Medicine 76 (2016) 1582-1593.

[40] E. Kellner, B. Dhital, V. G. Kiselev, M. Reisert, Gibbs-ringing artifact removal based on local subvoxelshifts, Magnetic Resonance in Medicine 76 (2016) 1574-1581.

[41] J. Andersson, S. Sotiropoulos, An integrated approach to correction for off-resonance effects and subject movement in diffusion MR imaging, Neuroimage 125 (2016) 1063-1078.

[42] S. Smith, Fast robust automated brain extraction, Human Brain Mapping 17 (2002) 143-155.

[43] A. Pavilla, G. Gambarota, A. Arrigo, M. Mejdoubi, R. Duvauferrier, H. Saint-Jalmes, Diffusional kurtosis imaging (DKI) incorporation into an intravoxel incoherent motion (IVIM) MR model to measure cerebral hypoperfusion induced by hyperventilation challenge in healthy subjects., Magnetic Resonance Materials in Physics, Biology and Medicine 30 (2017) 545-554.

[44] A. S. Rydhög, F. Szczepankiewicz, R. Wirestam, A. Ahlgren, C. F. Westin, L. Knutsson, O. Pasternak, Separating blood and water: Perfusion and free water elimination from diffusion MRI in the human brain, Neuroimage 156 (2017) 423-434.

[45] W. C. Wu, Y. F. Chen, H. M. Tseng, S. C. Yang, P. C. My, Caveat of measuring perfusion indexes using intravoxel incoherent motion magnetic resonance imaging in the human brain, European Radiology 25 (2015) 2485-2492.

[46] W. C. Wu, S. C. Yang, Y. F. Chen, H. M. Tseng, P. C. My, Simultaneous assessment of cerebral blood volume and diffusion heterogeneity using hybrid IVIM and DK MR imaging: initial experience with brain tumors, European Radiology 27 (2017) 306-314.

[47] R. H. Byrd, J. C. Gilbert, J. Nocedal, A trust region method based on interior point techniques for nonlinear programming, Mathematical Programming 89 (2000) 149-185.

[48] O. Gustafsson, M. Montelius, G. Starck, M. Ljungberg, An assessment of bayesian ivim model fitting, Proc. Intl. Soc. Magn. Reson. Med. 24 (2016) 2080.

[49] E. M. Meeus, J. Novak, S. B. Withey, N. Zarinabad, H. Dehghani, A. C. Peet, Evaluation of intravoxel incoherent motion fitting methods in low-perfused tissue, Journal of Magnetic Resonance Imaging 45 (2017) 1325-1334.

[50] O. Jalnefjord, M. Andersson, M. Montelius, G. Starck, A. K. Elf, V. Johanson, J. Svensson, M. Ljungberg, Comparison of methods for estimation of the intravoxel incoherent motion (IVIM) diffusion coefficient (D) and perfusion fraction (f), Magnetic Resonance Materials in Physics, Biology and Medicine (2018) doi: 10.1007/s10334-018-0697-5.

[51] O. J. Gurney-Champion, R. Klaassen, M. Froeling, S. Barbieri, J. Stoker, M. R. W. Engelbrecht, J. W. Wilmink, M. G. Besselink, A. Bel, H. W. M. van Laarhoven, A. J. Nederveen, Comparison of six fit algorithms for the intra-voxel incoherent motion model of diffusion-weighted magnetic resonance imaging data of pancreatic cancer patients, PLOS One 13 (2018) e0194590.

[52] P. T. While, A comparative simulation study of bayesian fitting approaches to intravoxel incoherent motion modeling in diffusion-weighted MRI, Magnetic Resonance in Medicine 78 (2017) 2373-2387.

[53] M. Jenkinson, P. Bannister, J. Brady, S. Smith, Improved optimisation for the robust and accurate linear registration and motion correction of brain images, NeuroImage 17 (2002) 825-841. 
[54] S. M. Smith, M. Jenkinson, M. W. Woolrich, C. F. Beckmann, T. E. J. Behrens, H. Johansen-Berg, P. R. Bannister, M. D. Luca, I. Drobnjak, D. E. Flitney, R. Niazy, J. Saunders, J. Vickers, Y. Zhang, N. D. Stefano, J. M. Brady, P. M. Matthews, Advances in functional and structural mr image analysis and implementation as FSL, NeuroImage 23 (2004) 208-219.

[55] M. S. Vinding, B. Guerin, T. Vosegaard, N. C. Nielsen, Local SAR, global SAR, and power-constrained large-flip-angle pulses with optimal control and virtual observation points, Magnetic Resonance in Medicine 77 (2017) 374-384.

[56] S. N. Jespersen, J. L. Olesen, A. Ianus, N. Shemesh, Implications of nongaussian diffusion on the interpretation of multidimensional diffusion measurements, Proc. Intl. Soc. Magn. Reson. Med. 26 (2018) 0886

[57] S. Takahashi, Neurovascular Imaging, MRI \& Microangiography, Springer-Verlag London Limited.

[58] T. H. Jochimsen, D. Ivanov, D. V. Ott, W. Heinke, R. Turner, H. E. Möller, J. R. Reichenbach, Wholebrain mapping of venous vessel size in humans using the hypercapnia-induced BOLD effect, NeuroImage 51 (2010) 765-774.

[59] F. Sepehrband, R. P. Cabeen, J. Choupan, G. Barisano, M. Law, A. W. Toga, the Alzheimers Disease Neuroimaging Initiative, A systematic bias in DTI findings, bioRxiv (2018) doi.org/10.1101/395012.

[60] J. Karbowski, Scaling of brain metabolism and blood flow in relation to capillary and neural scaling, PLOS One 6 (2011) e26709.

[61] I. I. Maximov, H. Thönneen, K. Konrad, L. Amort, I. Neuner, N. J. Shah, Statistical instability of TBSS analysis based on DTI fitting algorithm, Journal of Neuroimaging 25 (2015) 883-891.

[62] C. Federau, K. OBrien, A. Birbaumer, R. Meuli1, P. Hagmann1, P. Maeder, Functional mapping of the human visual cortex with intravoxel incoherent motion MRI, PLOS One 10 (2015) e0117706.

[63] M. Asgari, D. de Zelicourt, V. Kurtcuoglu, Glymphatic solute transport does not require bulk flow, Scientific Reports 6 (2016) 38635.

[64] I. I. Maximov, M. S. Vinding, D. H. Y. Tse, N. C. Nielsen, N. J. Shah, Real-time 2D spatially selective MRI experiments: Comparative analysis of optimal control design methods, Journal of Magnetic Resonance 254 (2015) 110-120.

[65] M. S. Vinding, D. Brenner, D. H. Y. Tse, S. Vellmer, T. Vosegaard, D. Suter, T. Stöcker, I. I. Maximov, Application of the limited-memory quasi-newton algorithm for multi-dimensional, large flip-angle RF pulses at 7T, Magnetic Resonance Materials in Physics, Biology and Medicine 30 (2017) 29-39. 